\title{
Oxymatrine Improves the Integrity of the Blood-brain Barrier After Cerebral Ischemia-reperfusion Injury Through the Caveolin-1/MMP-9 Signaling Pathway
}

\section{Jiaoyan $\mathrm{Yu}$}

Department of Pharmacy, Tangdu Hospital, Fourth Military Medical University

Qingqing Lu

Department of Pharmacy, Tangdu Hospital, Fourth Military Medical University

Xi Li

Department of Pharmacy, Tangdu Hospital, Fourth Military Medical University

Mei Zhao

Department of Pharmacy, Tangdu Hospital, Fourth Military Medical University

\section{Ting Sun}

Department of Pharmacy, Tangdu Hospital, Fourth Military Medical University

$\mathrm{Na} \mathrm{Hu}$

Department of Pharmacy, Tangdu Hospital, Fourth Military Medical University

\section{Wei Jiang}

Department of Pharmacy, Tangdu Hospital, Fourth Military Medical University

\section{Ruitao Zhang}

Department of Pharmacy, Tangdu Hospital, Fourth Military Medical University

\section{Peng Yang}

Department of Pharmacy, Tangdu Hospital, Fourth Military Medical University qi yang ( $\square$ yangqifmmu@126.com )

Xi'an Tangdu Hospital of No4 Military Medical University

\section{Research}

Keywords: Oxymatrine, Blood-brain barrier, Ischemia-reperfusion injury, Caveolin-1, MMP-9

Posted Date: October 29th, 2020

DOI: https://doi.org/10.21203/rs.3.rs-98095/v1

License: (1) (1) This work is licensed under a Creative Commons Attribution 4.0 International License. Read Full License 


\section{Abstract}

Ischemic stroke (IS) is a major neurological disease worldwide and is associated with extremely high morbidity and mortality. Oxymatrine (OMT) has neuroprotective properties and protects against IS. However, whether its protective effect involves the blood-brain barrier (BBB) integrity is unknown. In this study, we used in vivo and in vitro models of IS to evaluate the protective effect of OMT and its mechanism with regard to the BBB. Our results showed that OMT significantly improved the neurological function and brain state and reduced BBB permeability in a mouse model of cerebral ischemiareperfusion. Additionally, OMT improved the tight junction of bEend. 3 cells under oxygen-glucose deprivation. Moreover, intracranial lentivirus injection-induced Cav-1 knockdown reduced the neuroprotective effects of OMT. Our results indicated that OMT could improve cl/R injury-induced damage to the BBB, and its effects may be related to the regulation of the Cav-1/MMP-9 signaling pathway. This suggests that OMT may offer effective protection against BBB injury after $\mathrm{cl} / \mathrm{R}$.

\section{Introduction}

Stroke is a common disease of the nervous system with high morbidity, disability, and mortality, manifesting as an ischemic or hemorrhagic stroke. Ischemic stroke (IS) accounts for approximately 70$80 \%$ of the total number of stroke events and is the second most fatal disease worldwide [1]. Currently, intravenous thrombolysis and mechanical thrombectomy, the main treatments for IS, offer narrow treatment windows and application ranges and do not benefit most stroke patients [2]. Many clinical trials fail due to reduced neuroprotective effects in severely damaged blood vessels after IS [3]. A new research model of neuroprotection, consisting of neurons, vascular endothelial cells, astrocytes and phagocytes-neurovascular unit (NUV) [4], which focuses on the interaction between neurons, astrocytes, and capillaries, creating a new paradigm to understand the pathophysiology and drug development of stroke [5].As an essential part of the NVU, the BBB plays a vital role in IS pathophysiology Oxymatrine (OMT) is a quinazolidine alkaloid extracted from the traditional Chinese medicinal plant, Sophora flavesicum (kushen), which has various pharmacological properties [6].OMT helps treat cancer, arthritis, chronic hepatitis, and other diseases [7-9], and can play a neuroprotective role in animal models of cerebral ischemia-reperfusion (cl/R) injury [10-11]. Studies have shown that OMT can battle against ischemic and hypoxic brain damage in rats caused by apoptosis and oxidative stress [12]. Furthermore, OMT reportedly reduces hypoxic-ischemic brain injury in neonates by increasing the antioxidant enzyme activity, inhibiting cell death [13], and playing a protective role in cerebral ischemia/reperfusion injury. Thus, OMT was supposed as a neuroprotective agent by modulating various signaling pathways related to inflammatory cascade reaction secondary to cerebral ischemia. However, it is still unclear whether OMT has a protective effect on BBB after $\mathrm{cl} / \mathrm{R}$.

In this study, we used middle cerebral artery occlusion (MCAO) model mice and bEnd.3 cell line to confirm OMT protective effects on BBB failure after $\mathrm{cl} / \mathrm{R}$ injury and discovered that OMT elicits this effect through the Caveolin-1/MMP-9 pathway. 


\section{Materials And Methods}

\section{Animal studies}

Six to eight-week-old C57BL/6 mice purchased from the Experimental Animal Center of the Fourth Military Medical University were randomly divided into six animals per cage and housed at $24 \pm 2{ }^{\circ} \mathrm{C}$, relative humidity $50-60 \%$, and a $12 \mathrm{~h}$ light-dark cycle. All animals had free access to food and water. All mice were acclimatized in the laboratory environment for at least one week.

Mice were randomly divided into five groups ( $\mathrm{n}=8$ in each group): the control group, model group (I/R), OMT (LOT:19111302, Shanghai Pure Excellent Biology) low-dose group (1 mg/kg), OMT medium-dose group (10 mg/kg), and OMT high-dose group (100 mg/kg). OMT was intraperitoneally administered to the mice in the drug administration groups $30 \mathrm{~min}$ after the MCAO operation, the mice were then anesthetized with pentobarbital sodium $(50 \mathrm{mg} / \mathrm{kg}) 24 \mathrm{~h}$ after reperfusion, and their brain tissue was collected for subsequent experiments.

\section{Middle Cerebral Artery Occlusion (MCAO) Model}

To establish the MCAO model, mice were prepared by suture embolization, as described previously [14]. Briefly, the mice were anesthetized by administering an intraperitoneal injection of pentobarbital sodium (1\%), held in a supine position, and a 1-cm midline incision was made along the neck. The left common carotid artery (CCA), the external carotid artery (ECA), and the internal carotid artery (ICA) were identified and carefully dissected free from the surrounding nerves and fascia. A cable was attached to the distal end of the CCA and ECA. A temporary clip was placed on the ICA along with the arteriole, near the heart, and then ligated with the CCA. A small opening was then cut into the ECA, and an anchored line (MSMC21B120PK50, RWD Life Science) was inserted into the CCA, and the ECA was cut to insert the anchored line into the ICA. The line plug was inserted at approximately $10 \mathrm{~mm}$ from the fork mouth and fastened with the ECA, after which the CCA telecentric end thread was loosened and a surgical suture was performed around the ECA stump. The sham group was treated the same way but without a plug. Throughout the procedure, the mouse body temperature was maintained at $37^{\circ} \mathrm{C}$. An hour and a half after the procedure, the line plug was pulled out to allow the brain tissue reperfusion. Brain samples were collected $24 \mathrm{~h}$ after reperfusion. Blood flow was monitored in the MCAO mice using a laser speckle Doppler flowmeter (RFLSI III, RWD Life Science). Mice with blood flow reduction $<30 \%$ and/or those with an mNSS score of fewer than 7 points were excluded.

\section{Neurological Score}

The improved mNSS scoring system was used for the neurological scoring test $24 \mathrm{~h}$ after reperfusion. The evaluation included five tests, including the tail suspension test, platform crawling test, sensory test, bar balance test, reflex test, and abnormal movement test. The highest possible score was 18[15].

\section{Evaluation of Brain Infarct Volume}


Twenty-four hours after reperfusion, the mice were sacrificed, and brain tissue was immediately harvested. After a $20 \mathrm{~min}$ incubation at $-20^{\circ} \mathrm{C}$, the brain was placed in the brain slot of a vibratome and sliced into uniform $2 \mathrm{~mm}$-thick slices from the bifurcation of the miter joint. The brain tissue sections were dyed at $37^{\circ} \mathrm{C}$ for $30 \mathrm{~min}$ in $2 \%$ triphenyltetrazolium chloride (TTC) and fixed with $4 \%$ paraformaldehyde for $24 \mathrm{~h}$. Digital images of the brain were then taken, and the cerebral infarction area was calculated. The infarct was observed as a white region while the normal brain had a red color. The infarct area was measured using Image J.

\section{Nissl Staining}

As determined by the neurological score, mice that showed neural defects were perfused with normal saline and fixed with $4 \%$ paraformaldehyde. Their brains were then resected and fixed in $4 \%$ paraformaldehyde for $4 \mathrm{~h}$ at $4{ }^{\circ} \mathrm{C}$, then dehydrated by passing through a $20 \%$ sucrose solution and then a $30 \%$ sucrose solution $24 \mathrm{~h}$. The brain was then cut into twenty-five thick cerebral slices containing hippocampal and cortical tissues using a cryotome and placed directly on an adhesive slide. The sections were then stained with $0.1 \%$ tar purple dye for $10 \mathrm{~min}$ and then washed several times with clean water. The sections were then dehydrated with a $75 \%, 95 \%$, and $100 \%$ ethanol gradient (1 $\mathrm{m}$ in each solution). Finally, the sections were placed in xylene for $10 \mathrm{~min}$ and then mounted on a neutral gum slide. The sections were then observed under a high-powered bright-field microscope (Nikon Ni), and digital images were obtained. Cells with distinct nucleoli were identified as intact and undamaged cells.

\section{TUNEL Staining}

After fixing the mouse brain with $4 \%$ paraformaldehyde solution and gradient dehydration, the sections were brought to room temperature. Next, the sections were incubated with a solution containing $0.2 \%$ $\mathrm{H} 2 \mathrm{O} 2$ and methanol for $0.5 \mathrm{~h}$ to prevent endogenous peroxidase activity. They were then treated with the TUNEL reaction mixture (Roche) at $37^{\circ} \mathrm{C}$ for $60 \mathrm{~min}$. The TUNEL positive and negative cells were counted by fluorescence microscopy (OLYMPUS IX 53 + DP 73). The result is expressed as an apoptosis index, calculated as follows: (TUNEL positive cells)/(total cells) $\times 100 \%$.

\section{Evans Blue Leakage Detection}

The permeability of BBB was determined using the Evans blue dye. Mice were administered $2 \%$ Evans blue by the tail vein injection $(2 \mathrm{~mL} / \mathrm{kg}) 90$ min before sacrifice. After resecting the brain, it was weighed and homogenized in 1:3 (W/V) 50\% trichloroacetic acid (TCA) and was centrifuged (12000 × g, $15 \mathrm{~min}$ ). The supernatant was then used for the Evans blue colorimetric assay. The supernatant absorbance was determined at $620 \mathrm{~nm}$ using a microplate spectrophotometer to calculate the Evans blue transmittance.

\section{Determination of Brain Water Content}

Brain specimens were collected $24 \mathrm{~h}$ after reperfusion, and the cerebellum and hydrangea were removed, and the ischemic hemispheres were taken and weighed immediately (wet weight). They were then dried in an oven at $105^{\circ} \mathrm{C}$ for $72 \mathrm{~h}$ and then weighed (dry weight). The percentage of brain water content was calculated as [(wet weight - dry weight)/ wet weight $] \times 100 \%$. 


\section{Cell Culture}

Mouse brain microvascular endothelial cell line, bEnd.3, was obtained from Sixin Biotechnology, Shanghai, China, and maintained in endothelial cell complete medium at $37^{\circ} \mathrm{C}$ and $5 \% \mathrm{CO}_{2}$. Dulbecco's Modified Eagle Medium (DMEM) (AF29498406, HyClone) + 10\% fetal bovine serum (2206993CP, Gibco) + $1 \%$ penicillin and streptomycin mixture (20191028, Solarbio), The culture medium was changed every 2 days.

\section{Oxygen Glucose Deprivation (OGD) Model}

To simulate ischemic/reperfusion conditions in vitro, bEnd. 3 cells were reperfused after oxyglucose deprivation. Briefly, bEnd. 3 cells were washed three times with phosphate-buffered saline (PBS), and the normal medium was replaced with DMEM without glucose (2120596, Gibco). The cells were then transferred to a hypoxic chamber (95\% N2 / 5\% CO2, MIC-101, USA, Billups-Rothenberg) and incubated at $37^{\circ} \mathrm{C}$ for $2 \mathrm{~h}$. They were then cultured for another $24 \mathrm{~h}$ under standard culture conditions.

\section{Cell Counting Kit-8 (CCK-8) Assay}

Cell viability was measured using the CCK-8 assay. Cells $\left(4 \times 10^{3}\right)$ were plated in each well of a 96-well plate. After $24 \mathrm{~h}$ of pre-culture in the incubator, $10 \mu \mathrm{L}$ CCK-8 reagent (BS350B, Biosharop) was added to each well after OGD modeling and administration. After incubation in the incubator for $1 \mathrm{~h}$, the absorbance value at $490 \mathrm{~nm}$ was determined using a microplate reader. The absorbance was normalized to a reagent blank to calculate the cell viability.

\section{Annexin-V/PI double staining Assay}

Apoptosis was detected by Annexin-V/PI double staining. Briefly, $1 \times 10^{6}$ cells were plated in each well of a 6-well plate. Cells were harvested and incubated with Annexin-V-FITC $(5 \mu \mathrm{L})$ in the dark for 15 minutes at room temperature, after which $5 \mu \mathrm{L}$ PI staining solution was added and incubated for $5 \mathrm{~min}$. After adding $200 \mu \mathrm{L}$ Binding Buffer, the cell fluorescence was detected using flow cytometry immediately. The number of cells positive for Annexin-V-FITC and PI was used to calculate the apoptosis rate.

\section{Transendothelial Electrical Resistance (TEER)}

TEER values were measured using a Millicell-ers cell resistor (Millipore, USA) equipped with chopstick electrodes to assess the integrity of the bEnd. 3 monolayer cell barrier. Briefly, $2 \times 10^{5}$ cells were plated in the upper chamber of a transwell insertion chamber (3460; Corning, NY, USA) in $0.5 \mathrm{~mL}$ DMEM, and $1.5 \mathrm{ml}$ of the same medium was added to the lower chamber to prevent the formation of a pressure gradient. Studies have shown that bEnd. 3 cells have the highest TEER value on the 7th day, so this time point was selected to detect the TEER value. The TEER value $\left(\Omega \mathrm{cm}^{2}\right)$ was calculated after subtracting the resistance value of the blank transwell culture chamber without cells and multiplying it with the area of the upper culture chamber $\left(1.13 \mathrm{~cm}^{2}\right)$.

\section{FITC-dextran Permeability Test}


After $1.5 \mathrm{~h}$ of OGD followed by $24 \mathrm{~h}$ of reoxygenation, the culture medium in the upper and lower chambers of the transwell was replaced with a culture medium free of nutritional factors in preparation

for the permeation experiment. During the penetration experiment, FITC-Dextran (MW 1000, $10 \mathrm{kDa}$ ) was added to the transwell upper chamber at a $1 \mathrm{mg} / \mathrm{kg}$ concentration in DMEM. After incubating for $30 \mathrm{~min}$, $100 \mu \mathrm{l}$ of the media from the lower chamber was collected, and the fluorescence intensity was detected using a VICTOR Nivo multimode plate reader (PerkinElmer, UK) at an excitation/emission of 490/520 nm.

\section{Lentiviral constructs and shRNA for Cav-1}

Cav-1 lentivirus vectors with a GFP tag were constructed by Genepharma (Shanghai, China). To generate the Cav-1 short hairpin RNA (shRNA),the target sequence was designed against mouse Cav-1:

AAGGAGATTGACCTGGTCAAC 32.Microinjection of lentivirus into the lateral ventricle of the mouse brain interferes with the expression of Cav-1. Briefly, the mice were anesthetized by intraperitoneally injecting $1 \%$ sodium pentobarbital. The anesthetized mouse was placed in a stereotaxic device, the head hair shaved to expose the scalp, and an incision was made. The front chimney point of the mouse head was identified, and the coordinates were recorded. The position of the lateral ventricle $(0.3 \mathrm{~mm}$ behind the front chimney point, $1.0 \mathrm{~mm}$ left and right, depth: $2.2 \mathrm{~mm}$ ) was then estimated. A fine hole was then drilled in the skull in the bilateral ventricle area, and the needle was placed into the bilateral ventricle. The needle was allowed to rest for 10 min, after which $2 \mu$ lentivirus was slowly injected using a microsyringe at a speed of $0.2 \mu \mathrm{l} / \mathrm{min}$. After the injection, the needle remained in place for $10 \mathrm{~min}$, and then slowly pulled out. The scalp was sutured, and an anti-bacterial ointment was applied to prevent infection.

\section{Western Blotting Analysis}

Western blot was used to detect the protein expressions of CAV-1, MMP-9, MMP-2, occludin, ZO-1, P-gp, AQP-4, and occludin. Briefly, the brain tissue was homogenized in a lysis buffer containing protease inhibitor and phosphatase inhibitors. The total protein content was determined using a BCA protein assay. Equal amounts of proteins were resolved by SDS-PAGE and then transferred onto a PVDF membrane (Invitrogen, Carlsbad, California, USA). The membrane was blocked with 5\% skim milk/TBST $(\mathrm{pH} 7.6)$ for $1 \mathrm{~h}$. The membrane was then incubated with the primary antibodies - CAV-1 (ab2019, Abcam, 1:1000), MMP-9 (ab38898, Abcam, 1:1000), MMP-2 (10373-2-AP, Proteintech, 1:1000), occludin (13409-1-AP, Proteintech, 1:1000), ZO-1 (21773-1-AP, Proteintech, 1:1000), P-gp (ab170904, Abcam, 1:1000), AQP-4 (ab46182, Abcam, 1:1000), or actin (059M4770v, Sigma, 1:10000) - at $4{ }^{\circ} \mathrm{C}$ overnight. After incubation, the membrane was washed three times with TBST buffer and incubated with the corresponding secondary antibody $(1: 10,000)$. After incubation at room temperature for 1 hour, the membrane was washed three times with TBST. Protein bands were visualized under a chemiluminescence imager. The bands were quantified using Image $\mathrm{J}$, and the relative band density to $\beta$ actin was calculated[16]. The control ratio was set at $100 \%$, and the intensity of the treatment groups was expressed as a percentage relative to the control group.

\section{Statistical Analysis}


All results are expressed as mean \pm SD, and statistical analysis was conducted using Prism (GraphPad, San Diego, CA, USA) software. Two-tailed student's $t$-test was used for mean comparison between two groups, and one-way analysis of variance (ANOVA) was used for mean comparison between multiple groups (three groups or more). $\mathrm{P}<0.05$ was considered statistically significant.

\section{Results}

\section{OMT Reduced Infarction Volume and Improved Neurological Deficits}

To verify the I/R model success, the laser speckle Doppler imaging system was used to study blood flow in mice brains. The results showed that the blood flow after ischemia was reduced by about $30 \%$ compared with the blank group before modeling. Later, the blood flow recovered to about $70 \%$ (Fig. 1a), indicating that the brain I/R model was successful. To further confirm the OMT effect on a cl/R injury, mice were injected intraperitoneally with OMT (1 mg/kg, $10 \mathrm{mg} / \mathrm{kg}, 100 \mathrm{mg} / \mathrm{kg}) 30 \mathrm{~min}$ after modeling. TTC staining was performed to evaluate the infarct size, and a neurological severity score test (mNSS) was performed to determine the neurological effect. A higher mNSS score shows a lower neurological function. The mNSS score of the model group was the highest, and OMT treatment significantly debased the mNSS in a dose-dependent manner $(P<0.05)$ (Fig. 1b). TTC staining results showed that there was no infarct volume in the sham operation group, significantly increased in the model group, $(P<0.01)$. Moreover, the infarct volume was expectedly reduced after OMT treatment. Of note. these reductions were dose-dependent. $(P<0.05)$. (Fig. 1c-d).

\section{OMT Decreased Neuron Apoptosis After cl/R Injury}

In order to investigate the protective effect of OMT on neuronal morphology, Nissl staining and TUNEL staining were performed. As shown in the Fig. $2 \mathrm{a}$, in the sham operation group,no morphological changes were observed, the structure of neurons was clear, the arrangement was regular, and the density of neurons was not abnormal. However, the cortex and hippocampus of the I/R model group were damaged, and there was distinct nuclear pyknosis and glia, and cell swelling and interstitial edema were also observed. There were dissolution and necrosis of Nissl's central body in neurons. Compared to the model group, the OMT treated remarkably improved, the density definitely increased definitely (Fig. 2b-c). This finding suggests that OMT reduced neuron damage after I/R injury.

Of note, similar results were obtained with the TUNEL staining, in which 4',6-diamidino-2-phenylindole (DAPI) (blue fluorescence) marks the cell nuclei, while TUNEL positive cells are evidenced by red fluorescence. Firstly, the sham operation group had occasional red fluorescence compared with the sham operation group. Secondly, a large amount of red fluorescence in the I/R model group revealed an evident increase in apoptosis. Furthermore, the red fluorescent labeling was statistically significantly reduced after OMT treatment $(P<0.05)$ (Fig. 2d-e). From these results, we conclude that OMT inhibited neural apoptosis after $\mathrm{I} / \mathrm{R}$ injury. 


\section{OMT Ameliorated BBB Disruption in the $\mathrm{cl} / \mathrm{R}$ model}

To evaluate OMT protection of the BBB after I/R injury, Evans blue extravasation was used to monitor in vivo mice brain BBB permeability for large molecule leakage. The content of dye in the ischemia side was significantly lower in OMT groups compared to in the I/R group $(P<0.05)$, and the effect was dosedependent (Fig. 3a-b). The results of brain water content measurement were consistent with the above results. However, the sham operation group water content did not change compared with normal mice, while the I/R model group was significantly higher. OMT treatment counteracted this increase. (Fig. 3c). Therefore, we found that OMT treatment can reduce the permeability of the BBB in I/R model mice, thereby reducing brain edema.

\section{OMT Can Reduce BBB Permeability in the OGD Model}

To determine the protective effect of OMT on the BBB after brain I/R injury, The bEnd.3 cells were stained with the Annexin-V-FITC/PI dual staining kit and then detected by flow cytometry. Compared with the control group, the rate of apoptosis in the OGD group increased. However, OMT treatment can reverse the effect, and the treatment effect is dose-dependent. The apoptosis rate in the model group was significantly higher than that in the control group $(P<0.05)$, and the drug administration groups were significantly lower than that in the model group (Fig. 4a-b), suggesting that OMT can reduce cell apoptosis in the bEnd.3 OGD model. Next, the Cell Counting Kit-8 experiment was performed. The cell viability of the OGD group was significantly lower than that of the control group $(P<0.05)$. After OMT intervention, cell viability was improved in a dose-dependent manner, especially, the high-dose group viability as high as $80 \%$ of the OGD group (Fig. 4c).

To explore the OMT effect on the BBB integrity and permeability, we established a BBB model in vitro, and a FITC-dextran leakage test and TEER measurements were carried out (Figure. 4d-e). The model group showed significantly higher FITC-dextran penetration and lower TEER values. By contrast to the model group. after OMT treatment, the low-dose group had no effect, however, the medium-dose group and the high-dose group showed an apparent reduction in the FITC-dextran penetration and enhancement TEER values. Taken together,these results demonstrated that OMT could produce anti-apoptosis in vitro and improve the permeability and integrity of the BBB model in vitro.

\section{OMT Improved the Expression of BBB Related Proteins After cl/R Injury}

The TJ of vascular endothelial cells in the central nervous system is essential for the BBB integrity, AQP-4 is involved in the formation of brain edema after brain injury, and MMP-2 and transporter protein P-gp are involved in the brain injury process of ischemia-reperfusion [17]. In order to study at the molecular level whether OMT affects the integrity and permeability of the BBB after cerebral I/R injury, we use western blot analysis to detect changes in the expression of these proteins after $\mathrm{l} / \mathrm{R}$ modeling and drug administration. The results showed in the Fig. 5a, that the expression of TJ protein ZO-1 and Occludin 
was significantly reduced after modeling. Compared with the model group, OMT significantly reduced the loss of TJ proteins (Fig. 5b-c). In addition, OMT can resist the reduction of P-gp(Fig. 5d) and lower the expression of MMP-2(Fig. 5e) and AQP-4(Fig. 5f-g). Besides, These results verified that OMT could resist the changes in the integrity of the BBB and the expression of permeability-related proteins caused by $\mathrm{l} / \mathrm{R}$ injury, thereby protecting the brain.

\section{OMT reduced the Expression of Cav-1/MMP-9 Signaling Pathway Proteins After Cerebral I/R Injury}

The Cav-1/MMP-9 pathway is a key therapeutic target that can protect BBB integrity from cl/R [18]. To establish whether OMT can protect the brain after cerebral I/R by regulating the expression of Cav1/MMP-9 after BBB damage, western blot analysis was used to measure the expression of Cav-1 and MMP-9 protein after modeling and OMT administration. Further, Cav-1 expression was conspicuously increased in the model group and significantly decreased after OMT intervention in a dose-dependent manner(Fig. 6a-b). Moreover, the expression of MMP-9 protein showed an the identical variation trend with Cav-1 (Fig. 6c-d). Therefore,we argue that the BBB-protective effect of OMT might be mediated by the downregulated expression of Cav-1/MMP-9 pathway proteins.

\section{The Protective Effect of OMT Was Significantly Weakened After Cav-1 Was Knocked Down}

To deeply investigate whether OMT participates in the brain protection after cerebral I/R through the Cav1/MMP-9 pathway, we knocked down Cav-1 protein expression in mice and OMT protective effect. As can be seen from Fig. 7a-b, the expression of Cav-1 in the lentivirus interference group was significantly reduced, demonstrating that the virus interference was successful. The results of TTC staining showed that there was no significant difference in infarct size between the shCav-1 group and the OMT administration group (Fig. 7c-d). Similarly, no striking difference in the neurological scores was observed (Fig. 7e). In addition, OMT could not reduce the expression of MMP-9 and resist the loss of TJ protein (Fig. 7f-i). These results demonstrated that the brain protection effect of OMT depends on the participation of the Cav-1/MMP-9 pathway.

\section{Discussion}

Stroke is a life-threatening disease with high morbidity and mortality that causes permanent disabilities, characterized by multifaceted pathogenesis [19].Notably, IS may severely damage the NVU [20]. This study first proved that OMT protective effects not only act on neurons but also capillaries, glia, and extracellular matrix. We discovered that this protective effect might be achieved by regulating the Cav1/MMP-9 signaling pathway.

OMT is extracted from the traditional Chinese medicinal plant, initially reported to exert neuroprotective effects post-IS. Here, we assessed OMT's neurological function scores, TTC staining, Nissl staining and 
TUNEL staining. The blood flow in blood vessels decreases after stroke[21], as determined by laser speckle Doppler scanning. Based on our research group's preliminary research, it is proved that $100 \mathrm{mg} / \mathrm{kg}$ OMT has a neuroprotective effect [22], Although the OMT neuroprotective effect is relatively weak at low doses, it gradually, dose-dependently increases to achieve peak activity at $100 \mathrm{mg} / \mathrm{kg}$, consistently with previous reports[23][24].

BBB destruction is a common consequence of stroke, leading to severen complications, contributing to further development of cerebral ischemic injury[25]. BBB does not operate independently but as a multicellular neurovascular module of the NVU $[4,26]$. The BBB plays a pivot in the pathophysiology of IS. Currently, no previous report suggested the protective effect of OMT on BBB. Therefore, we focused on the effects of OMT on BBB integrity. Firstly, we demonstrated that OMT could reduce brain permeability by examined the water content, EB penetration after I/R injury. EB leakage test is commonly used in scientific research to observe the integrity of BBB[27], When the BBB of the nervous system is destroyed, EB can enter the nervous system and stain it. We further proved that OMT can reduce OGD-induced BBB damage in endothelial cells cultured in vitro, which showed that OMT administration increased the TEER value and reduced the FITC-dextran leakage in bEend. 3 cell line, TEER is a classic cell transmembrane resistance measurement experiment; the TEER value of ECs directly relates to para-EC lysis [28], routinely used to evaluate BBB integrity in vitro. The FITC-dextran leak test evaluates the permeability of the BBB in vitro [29]. Taken together, OMT can protect the integrity and permeability of the BBB under the condition of ischemia and hypoxia.

The core element that constitutes the BBB is the endothelial cells (ECs) of the capillaries and the TJ proteins between the ECs. This unique anatomical basis significantly limits the bypass transport and cross-cell transport of substances between cells. This is the main feature of BBB ECs that distinguish them from other tissues. When a stroke occurs, due to the loosening of the TJs of ECs, a large paracellular space is formed, allowing blood flow to penetrate the brain parenchyma easily and ultimately promote vasogenic edema. TJ proteins, including occludin, claudins, and the major scaffolding protein ZO-1, play a critical role in the BBB formation and integrity [30],When a stroke occurs, the BBB is damaged and the TJ protein is degraded[31]. Our results show that OMT can reduce the degradation of TJ proteins occludin and ZO-1. Aquaporin 4 (AQP4) is the most abundant water-permeable channel found in the mammalian brain. I/R injury may trigger AQP4 upregulation and contribute to edema formation [32]. In addition brain edema and AQP-4 expression were increased after IS, OMT can inhibit this increase and reduce cerebral edema. P-Glycoprotein ( $\mathrm{P}-\mathrm{gp}$ ) is a transporter protein overexpressed on the luminal membrane of brain microvessels and is considered an important $d$ drug transporter on the BBB [33]. After IS, the expression of P-gp will increase, OMT reduced the expression of P-gp and restored the transport function of BBB. In summary, OMT improved the integrity and permeability of the BBB at the molecular level

Caveolin-1 (Cav-1), the main structural protein of vesicles, has been shown to play an essential role in endocytosis, vesicle transport, and signal transduction, leading to BBB damage by blocking tight linker in various pathological conditions [34-35]. Matrix metalloproteinases (MMPs), such as MMP-2 and MMP-9, 
a family of zinc-binding proteolytic enzymes, are important in mediating BBB disruption after cerebral ischemia by degrading the major structural components [36]. Previous studies indicated that Cav-1 might interact with MMPs in a stimulus-dependent manner[37]. Studies have confirmed that MMP-9 can degrade TJ in cerebral ECs in vitro and MCAO rats, ascribed to the increased BBB permeability. Furthermore, Cav-1 reportedly regulates BBB permeability through extracellular and paracellular pathways in stroke. Besides, MMP-9 expression was regulated to affect TJ protein expression and aggravate the BBB damage after $\mathrm{cl} / \mathrm{R}$ [38-39]. In our study, we observed that the expression of Cav-1 in the I/R mouse model was significantly increased compared to that in the control group. OMT can reduce the expression of Cav-1 protein in model mice. We further found that OMT can resist the matrix after brain injury and attenuate the increase in MMP-9. Therefore, it is speculated that OMT may reduce the MMP-9 protein expression by downregulating the expression of Cav-1, thereby reducing the degradation of TJ protein. To further confirm these findings, we used a brain-localized injection of lentiviral interference to reduce mice Cav-1 protein expression. Western blot results showed that Cav-1 expression was significantly reduced compared with the negative control group, and the knockout was successful. Our results showed that the OMT administration did not attenuate MMP-9 expression and did not have a neuroprotective effect in the Cav-1 knockdown mice, suggesting the protective effect of OMT on the BBB after $\mathrm{cl} / \mathrm{R}$ injury depends on the Cav-1/MMP-9 signaling pathway.

In summary, our study proved that OMT protects the BBB after $\mathrm{cl} / \mathrm{R}$ in vivo, and protect the brain microvascular ECs after OGD injury in vitro. OMT reduces MMP-9 expression by modulating Cav-1 expression, thus maintains BBB integrity and the expression of permeability-related proteins. These findings partially reveal the molecular mechanism underlying the BBB-protective properties of OMT in I/R injury. A preliminary understanding of OMT protective effects on the BBB structure and function has been gained, and the mechanism of its action has been discussed. However, the specific function, regulation mode, and detailed molecular mechanisms under pathological conditions need to be further studied. Further studies will provide a deeper understanding of the mechanism underlying the BBB-protective effects of OMT, providing a more in-depth theoretical basis for clinical applications and the future treatment of ischemic cerebrovascular diseases.

\section{Abbreviations}

IS

ischemic stroke; cl/R:cerebral ischemia-reperfusion; OMT:oxymatrine; BBB:blood-brain barrier; I/R:ischemia-reperfusion; Cav-1:Caveolin-1; MCAO:middle cerebral artery occlusion; MMP-9:matrix metalloproteinase 9; TJ protein:tight junction protein; OGD:oxygen-glucose deprivation; EB:Evans blue; TEER:trans epithelial electric resistance; P-gp:p-glycoprotein; NVU:neurovascular unit; NC:negative control

\section{Declarations}

\section{Acknowledgements}


None

\section{Authors' contributions}

JJ-Y carried out the experiments. QY conceived and designed the study and revised the manuscript. Qingqing Lu and Xi Li helped to draft the manuscript. Mei Zhao and Ting Sun participated in the acquisition of data. $\mathrm{Na} \mathrm{Hu}$ and Wei Jiang carried out analysis and interpretation of data. Ruitao Zhang,Peng Yang, performed the statistical analysis. All authors read and approved the final manuscript.

\section{Funding}

This work was supported by National Natural Science Foundation Grants (nos. 81870893, to QY),and by the National Postdoctoral Program for Innovative Talents (grant no. BX20160025, to QY). and by Chinese Postdoctoral Science Foundatio (nos.45416)

\section{Availability of data and materials}

The datasets supporting the conclusion of this study are included within the article

\section{Ethics approval and consent to participate}

The animal procedures involved in this study were approved by the Animal Care and Use Committee of the Fourth Military Medical University

\section{Consent for publication}

Not applicable.

\section{Competing interests}

The authors declare that they have no competing interests.

\section{Author details}

${ }^{1}$ Precision Pharmacy \& Drug Development Center, Department of Pharmacy, Tangdu Hospital, Fourth Military Medical University, Xian 710038, China.

\section{References}

1. Wilkins SS, Bourke P, Salam A, Akhtar N, D'Souza A, Kamran S, Bhutta Z, Shuaib A: Functional Stroke Mimics: Incidence and Characteristics at a Primary Stroke Center in the Middle East. Psychosom Med 2018;80:416-421.

2. Dewar B, Shamy M: tPA for Acute Ischemic Stroke and Its Controversies: A Review. Neurohospitalist 2020;10:5-10. 
3. Yang Y, Yang LY, Orban L, Cuylear D, Thompson J, Simon B, Yang Y: Non-invasive vagus nerve stimulation reduces blood-brain barrier disruption in a rat model of ischemic stroke. Brain Stimul 2018;11:689-698.

4. Steliga A, Kowianski P, Czuba E, Waskow M, Morys J, Lietzau G: Neurovascular Unit as a Source of Ischemic Stroke Biomarkers-Limitations of Experimental Studies and Perspectives for Clinical Application. Transl Stroke Res 2020;11:553-579.

5. Campbell B, De Silva DA, Macleod MR, Coutts SB, Schwamm LH, Davis SM, Donnan GA: Ischaemic stroke. Nat Rev Dis Primers 2019;5:70.

6. Cao X, He Q: Anti-Tumor Activities of Bioactive Phytochemicals in Sophora flavescens for Breast Cancer. Cancer Manag Res 2020;12:1457-1467.

7. Halim CE, Xinjing SL, Fan L, Bailey VJ, Arfuso F, Tan CH, Narula AS, Kumar AP, Sethi G, Ahn KS: Anticancer effects of oxymatrine are mediated through multiple molecular mechanism(s) in tumor models. Pharmacol Res 2019;147:104327.

8. Gu LL, Shen ZL, Li YL, Bao YQ, Lu H: Oxymatrine Causes Hepatotoxicity by Promoting the Phosphorylation of JNK and Induction of Endoplasmic Reticulum Stress Mediated by ROS in LO2 Cells. Mol Cells 2018;41:401-412.

9. Jiang Y, Sang W, Wang C, Lu H, Zhang T, Wang Z, Liu Y, Xue B, Xue S, Cai Z, Hua Y, Zhu L, Ma J: Oxymatrine exerts protective effects on osteoarthritis via modulating chondrocyte homoeostasis and suppressing osteoclastogenesis. J Cell Mol Med 2018.

10. Dong XQ, Yu WH, Hu YY, Zhang ZY, Huang M: Oxymatrine reduces neuronal cell apoptosis by inhibiting Toll-like receptor 4/nuclear factor kappa-B-dependent inflammatory responses in traumatic rat brain injury. Inflamm Res 2011;60:533-539.

11. Zhou S, Qiao B, Chu X, Kong Q: Oxymatrine attenuates cognitive deficits through SIRT1-mediated autophagy in ischemic stroke. J Neuroimmunol 2018;323:136-142.

12. Liu Y, Wang H, Liu N, Du J, Lan X, Qi X, Zhuang C, Sun T, Li Y, Yu J: Oxymatrine protects neonatal rat against hypoxic-ischemic brain damage via PI3K/Akt/GSK3beta pathway. Life Sci 2020;254:116444.

13. Zhao P, Zhou R, Li HN, Yao WX, Qiao HQ, Wang SJ, Niu Y, Sun T, Li YX, Yu JQ: Oxymatrine attenuated hypoxic-ischemic brain damage in neonatal rats via improving antioxidant enzyme activities and inhibiting cell death. Neurochem Int 2015;89:17-27.

14. McBride DW, Zhang JH: Precision Stroke Animal Models: the Permanent MCAO Model Should Be the Primary Model, Not Transient MCAO. Transl Stroke Res 2017.

15. Han B, Zhang Y, Zhang Y, Bai Y, Chen X, Huang R, Wu F, Leng S, Chao J, Zhang JH, Hu G, Yao H: Novel insight into circular RNA HECTD1 in astrocyte activation via autophagy by targeting MIR142-TIPARP: implications for cerebral ischemic stroke. Autophagy 2018;14:1164-1184.

16. Luo L, Sun T, Yang L, Liu A, Liu QQ, Tian QQ, Wang Y, Zhao MG, Yang Q: Scopoletin ameliorates anxiety-like behaviors in complete Freund's adjuvant-induced mouse model. Mol Brain 2020;13:15.

17. Nikolenko VN, Oganesyan MV, Vovkogon AD, Nikitina AT, Sozonova EA, Kudryashova VA, Rizaeva NA, Cabezas R, Avila-Rodriguez M, Neganova ME, Mikhaleva LM, Bachurin SO, Somasundaram SG, 
Kirkland CE, Tarasov VV, Aliev G: Current Understanding of Central Nervous System Drainage Systems: Implications in the Context of Neurodegenerative Diseases. Curr Neuropharmacol 2019.

18. Jin $X$, Sun $Y, X u$ J, Liu W: Caveolin-1 mediates tissue plasminogen activator-induced MMP-9 upregulation in cultured brain microvascular endothelial cells. J Neurochem 2015;132:724-730.

19. Topcuoglu MA, Saka E, Silverman SB, Schwamm LH, Singhal AB: Recrudescence of Deficits After Stroke: Clinical and Imaging Phenotype, Triggers, and Risk Factors. JAMA Neurol 2017;74:10481055.

20. Cai W, Zhang K, Li P, Zhu L, Xu J, Yang B, Hu X, Lu Z, Chen J: Dysfunction of the neurovascular unit in ischemic stroke and neurodegenerative diseases: An aging effect. Ageing Res Rev 2017;34:77-87.

21. Casas Al, Geuss E, Kleikers P, Mencl S, Herrmann AM, Buendia I, Egea J, Meuth SG, Lopez MG, Kleinschnitz C, Schmidt H: NOX4-dependent neuronal autotoxicity and BBB breakdown explain the superior sensitivity of the brain to ischemic damage. Proc Natl Acad Sci U S A 2017;114:1231512320.

22. Zhang K, Li YJ, Yang Q, Gerile O, Yang L, Li XB, Guo YY, Zhang N, Feng B, Liu SB, Zhao MG: Neuroprotective effects of oxymatrine against excitotoxicity partially through down-regulation of NR2B-containing NMDA receptors. Phytomedicine 2013;20:343-350.

23. Mao J, Hu Y, Zhou A, Zheng B, Liu Y, Du Y, Li J, Lu J, Zhou P: Oxymatrine reduces neuroinflammation in rat brain: A signaling pathway. Neural Regen Res 2012;7:2333-2339.

24. Shi HJ, Song HB, Wang L, Xiao SX, Bo KP, Ma W: The synergy of diammonium glycyrrhizinate remarkably reduces the toxicity of oxymatrine in ICR mice. Biomed Pharmacother 2018;97:19-25.

25. Umlauf BJ, Shusta EV: Exploiting BBB disruption for the delivery of nanocarriers to the diseased CNS. Curr Opin Biotechnol 2019;60:146-152.

26. Su EJ, Cao C, Fredriksson L, Nilsson I, Stefanitsch C, Stevenson TK, Zhao J, Ragsdale M, Sun YY, Yepes M, Kuan CY, Eriksson U, Strickland DK, Lawrence DA, Zhang L: Microglial-mediated PDGF-CC activation increases cerebrovascular permeability during ischemic stroke. Acta Neuropathol 2017;134:585-604.

27. Goldim M, Della GA, Petronilho F: Using Evans Blue Dye to Determine Blood-Brain Barrier Integrity in Rodents. Curr Protoc Immunol 2019;126:e83.

28. Li Y, Wang R, Xue L, Yang Y, Zhi F: Astilbin protects against cerebral ischaemia/reperfusion injury by inhibiting cellular apoptosis and ROS-NLRP3 inflammasome axis activation. Int Immunopharmacol 2020;84:106571.

29. Hu S, Wu Y, Zhao B, Hu H, Zhu B, Sun Z, Li P, Du S: Panax notoginseng Saponins Protect Cerebral Microvascular Endothelial Cells against Oxygen-Glucose Deprivation/Reperfusion-Induced Barrier Dysfunction via Activation of PI3K/Akt/Nrf2 Antioxidant Signaling Pathway. Molecules 2018;23.

30. Li M, Wen Y, Zhang R, Xie F, Zhang G, Qin X: Adenoviral vector-induced silencing of RGMa attenuates blood-brain barrier dysfunction in a rat model of MCAO/reperfusion. Brain Res Bull 2018;142:54-62.

31. Knowland D, Arac A, Sekiguchi KJ, Hsu M, Lutz SE, Perrino J, Steinberg GK, Barres BA, Nimmerjahn A, Agalliu D: Stepwise recruitment of transcellular and paracellular pathways underlies blood-brain 
barrier breakdown in stroke. Neuron 2014;82:603-617.

32. Filchenko I, Blochet C, Buscemi L, Price M, Badaut J, Hirt L: Caveolin-1 Regulates Perivascular Aquaporin-4 Expression After Cerebral Ischemia. Front Cell Dev Biol 2020;8:371.

33. De Lange E, Vd BD, Bellanti F, Voskuyl RA, Syvanen S: P-glycoprotein protein expression versus functionality at the blood-brain barrier using immunohistochemistry, microdialysis and mathematical modeling. Eur J Pharm Sci 2018;124:61-70.

34. Gu Y, Zheng G, Xu M, Li Y, Chen X, Zhu W, Tong Y, Chung SK, Liu KJ, Shen J: Caveolin-1 regulates nitric oxide-mediated matrix metalloproteinases activity and blood-brain barrier permeability in focal cerebral ischemia and reperfusion injury. J Neurochem 2012;120:147-156.

35. Chen HS, Chen X, Li WT, Shen JG: Targeting RNS/caveolin-1/MMP signaling cascades to protect against cerebral ischemia-reperfusion injuries: potential application for drug discovery. Acta Pharmacol Sin 2018;39:669-682.

36. Yue KY, Zhang PR, Zheng MH, Cao XL, Cao Y, Zhang YZ, Zhang YF, Wu HN, Lu ZH, Liang L, Jiang XF, Han H: Neurons can upregulate Cav-1 to increase intake of endothelial cells-derived extracellular vesicles that attenuate apoptosis via miR-1290. Cell Death Dis 2019;10:869.

37. Huang Q, Zhong W, Hu Z, Tang X: A review of the role of cav-1 in neuropathology and neural recovery after ischemic stroke. J Neuroinflammation 2018;15:348.

38. Fu S, Gu Y, Jiang JQ, Chen X, Xu M, Chen X, Shen J: Calycosin-7-O-beta-D-glucoside regulates nitric oxide /caveolin-1/matrix metalloproteinases pathway and protects blood-brain barrier integrity in experimental cerebral ischemia-reperfusion injury. J Ethnopharmacol 2014;155:692-701.

39. Umesalma S, Houwen FK, Baumbach GL, Chan SL: Roles of Caveolin-1 in Angiotensin II-Induced Hypertrophy and Inward Remodeling of Cerebral Pial Arterioles. Hypertension 2016;67:623-629.

\section{Figures}


a

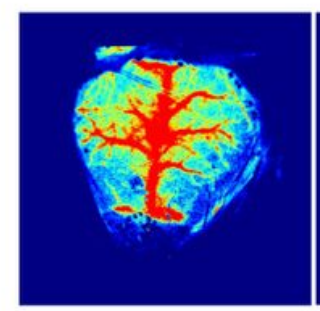

Before ischemia

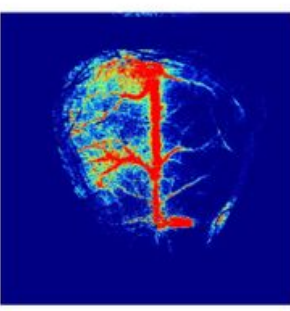

After ischemia

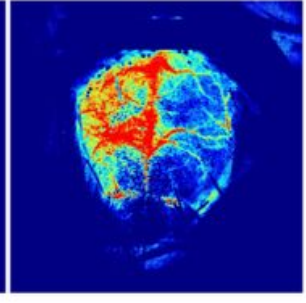

After reperfusion

\section{C}
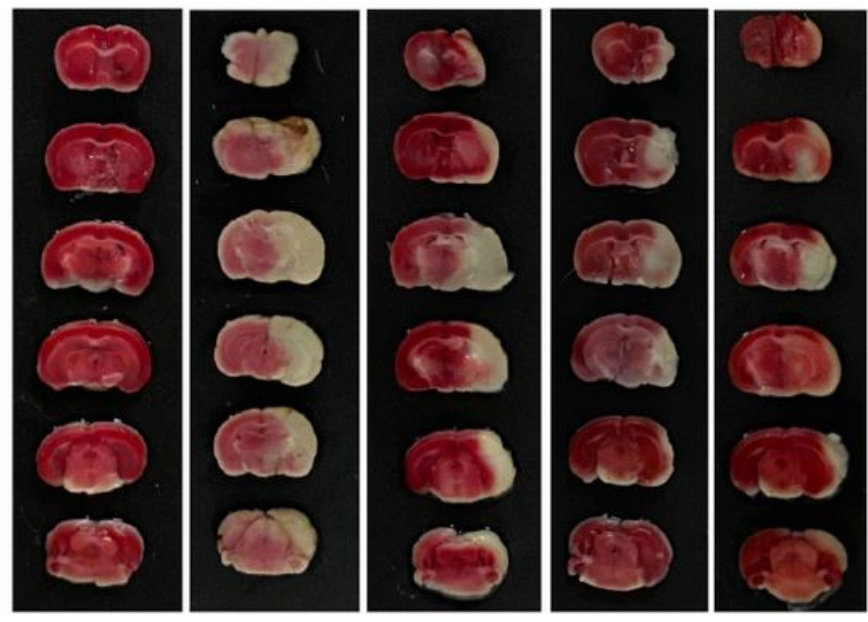

Sham

I/R

$1 \mathrm{mg} / \mathrm{kg}$

$10 \mathrm{mg} / \mathrm{kg} 100 \mathrm{mg} / \mathrm{kg}$

OMT

b
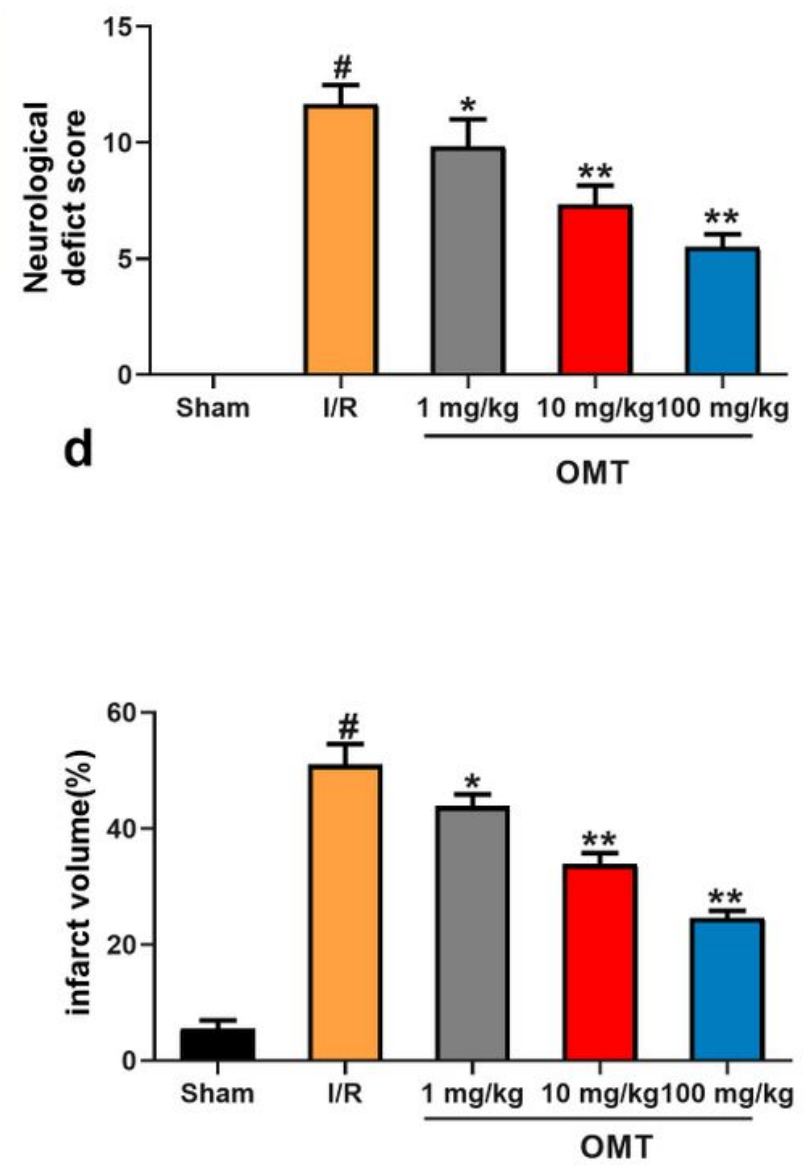

\section{Figure 1}

OMT application can reduce the cerebral infarction area and improve the nerve function defect in $\mathrm{I} / \mathrm{R}$ mice. (a) Laser speckle Doppler results showed that blood flow to the brain was blocked after MCAO. (b) Neurological function score of the model $24 \mathrm{~h}$ after I/R. (c) representative images of TTC stained brain sections in each group $24 \mathrm{~h}$ after cerebral I/R. (d) Quantitative results of the infarct area by TTC staining in each group. $n=8$ mice per group; $\# p<0.01$ versus sham group; ${ }^{*} p<0.01,{ }^{*} p<0.05$ versus I/R group 
a

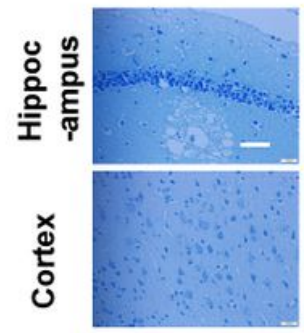

Sham

d
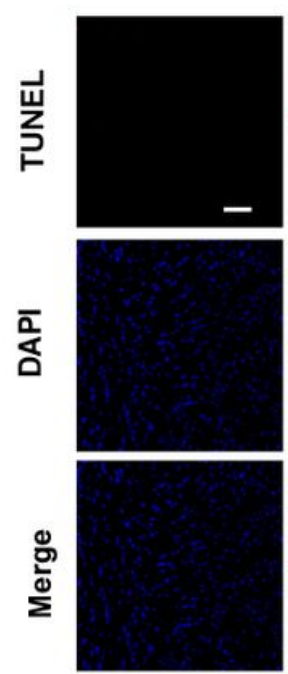

Sham

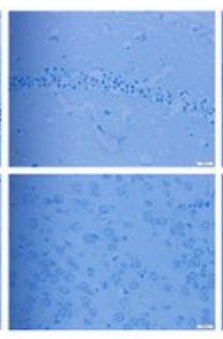

I/R
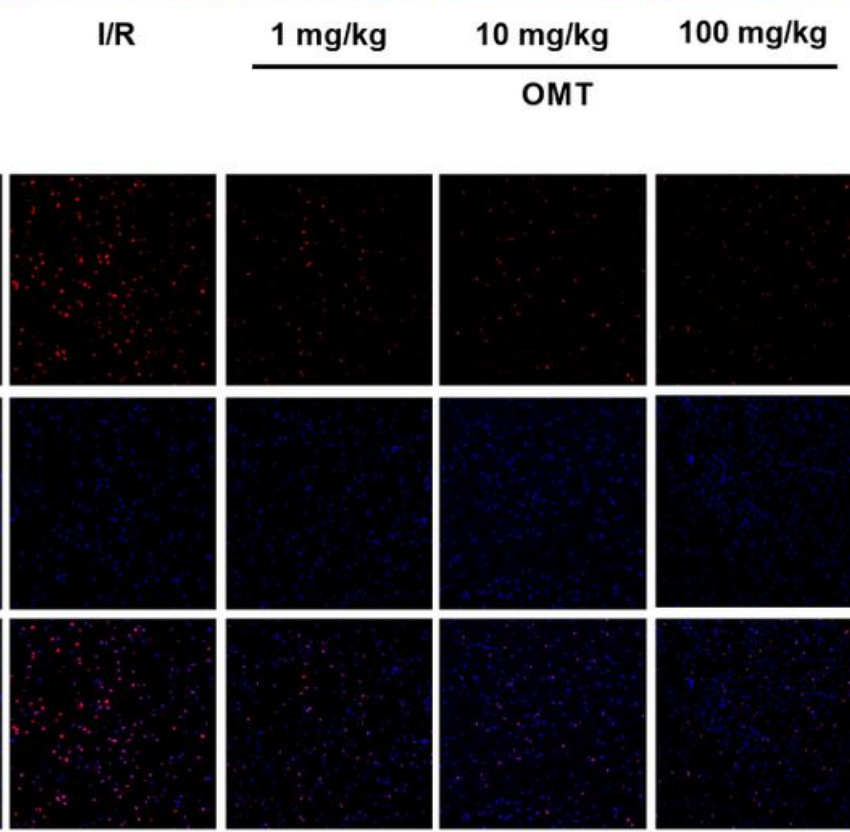

I/R
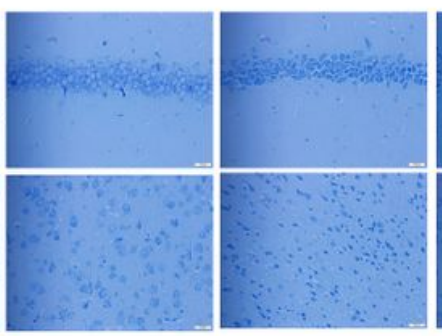

OMT
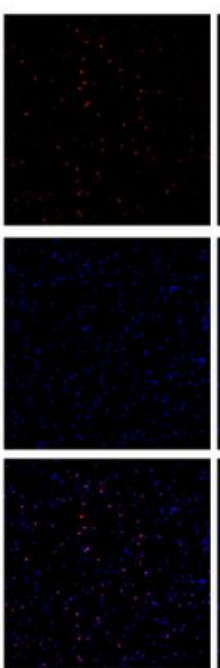

$1 \mathrm{mg} / \mathrm{kg}$
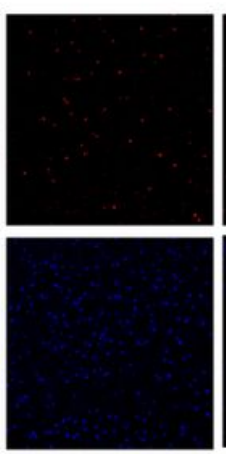
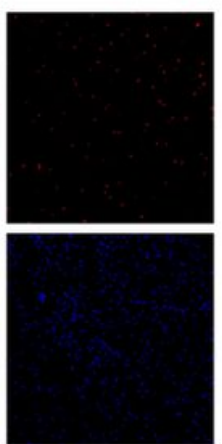

b

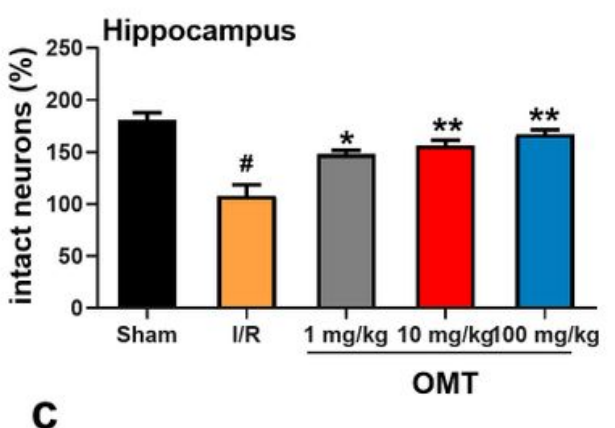

Cortiex

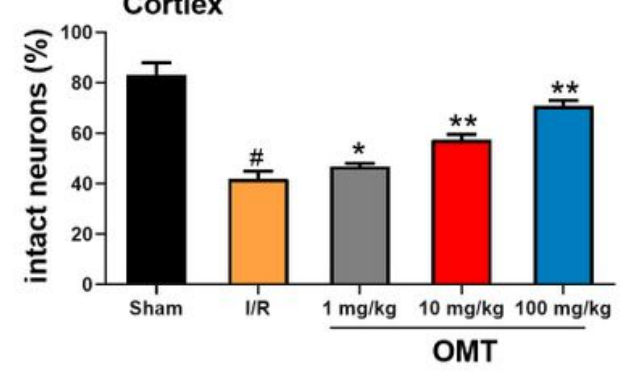

e

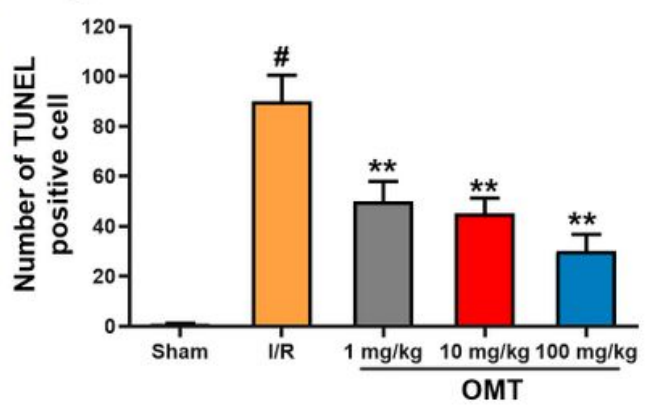

Figure 2

OMT treatment can reduce neuronal damage in I/R mice. (a)Nissl staining showing the morphological changes in neurons in the CA1 region of the cortex and hippocampus. (b-c) Statistical analysis of the percentage of intact neurons in the cortex and hippocampus CA1 area of female rats.Scale bar $=250 \mu \mathrm{m}$ (d) TUNEL staining was performed on the brain tissue sections of each group $24 \mathrm{~h}$ after modeling.Scale bar $=75 \mu \mathrm{m}(\mathrm{e})$ Numerical analysis of Nissl staining with image J program; $\mathrm{n}=8$ mice per group $\# \mathrm{p}<0.01$ versus sham group; ${ }^{\star \star} p<0.01,{ }^{\star} p<0.05$ versus $\mathrm{I} / \mathrm{R}$ group 
a

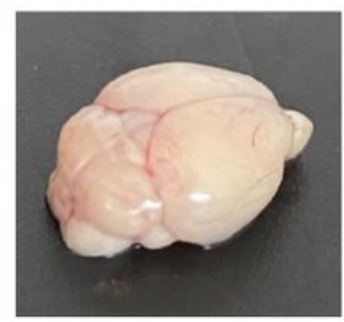

Sham

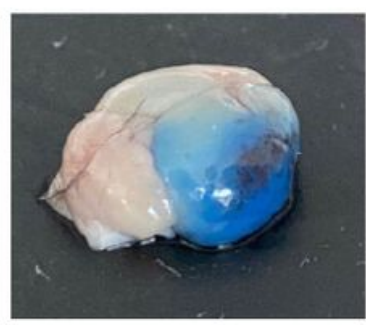

I/R

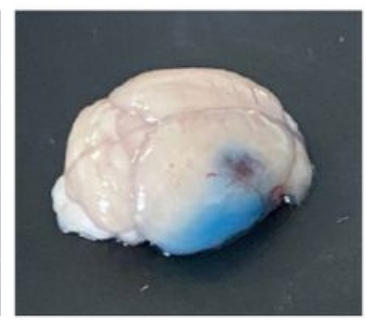

$1 \mathrm{mg} / \mathrm{kg}$

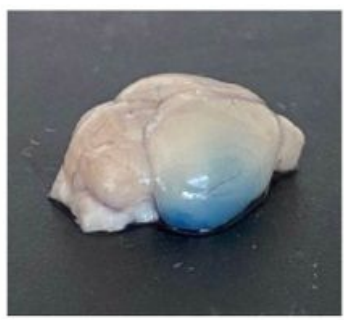

$10 \mathrm{mg} / \mathrm{kg}$

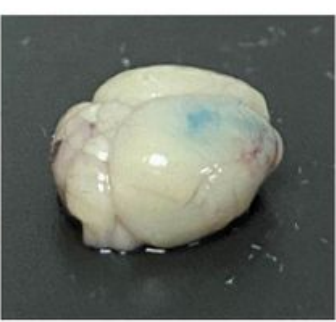

$100 \mathrm{mg} / \mathrm{kg}$ b

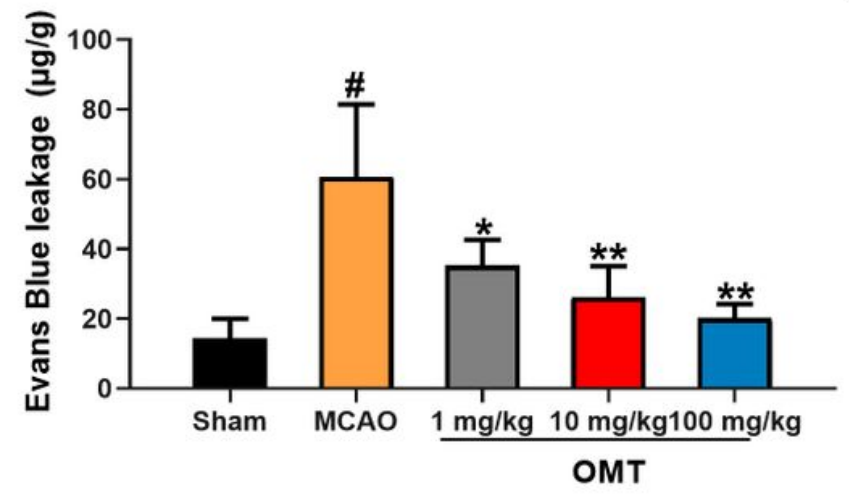

C

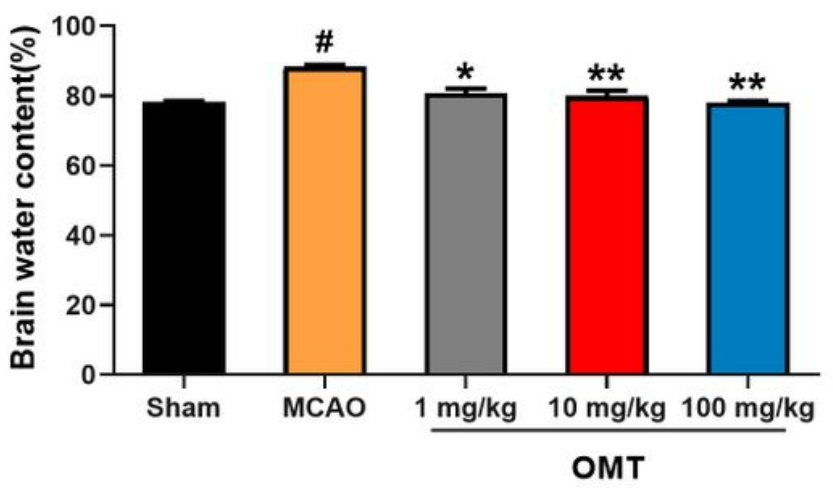

\section{Figure 3}

OMT can reduce brain edema and BBB leakage after I/R in vivo. (a) Representative Evans blue staining images $24 \mathrm{~h}$ after operation. (b) Quantitative analysis of Evans blue extravasation in the ipsilateral ischemic hemisphere of rats. (c) Determination of brain water content of ischemic hemisphere in each group; $\mathrm{n}=8$ mice per group. $\# \mathrm{p}<0.01$ versus sham group; ${ }^{* \star} \mathrm{p}<0.01,{ }^{*} \mathrm{p}<0.05$ versus $\mathrm{I} / \mathrm{R}$ group 
a

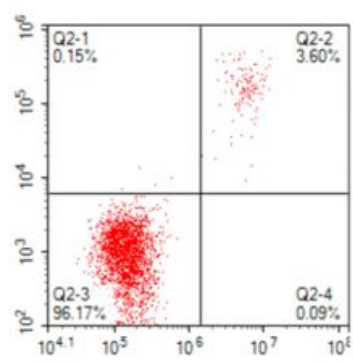

Control

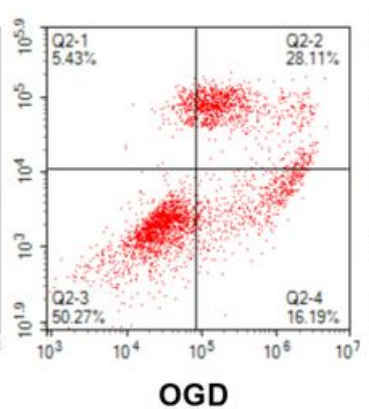

OGD

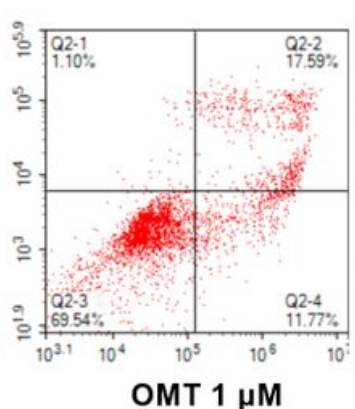

OMT $1 \mu \mathrm{M}$
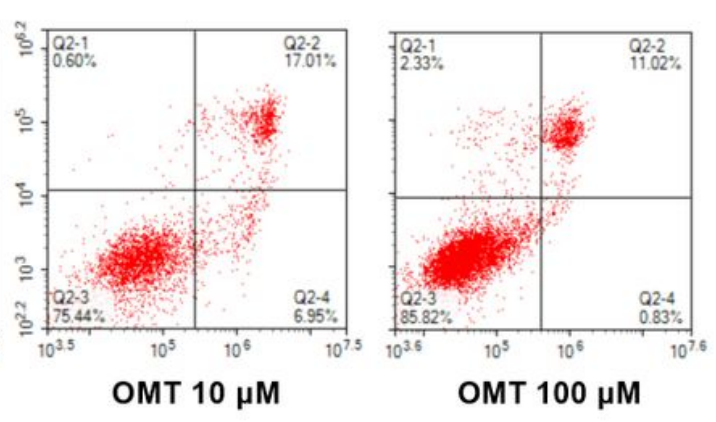

d

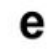

e

C

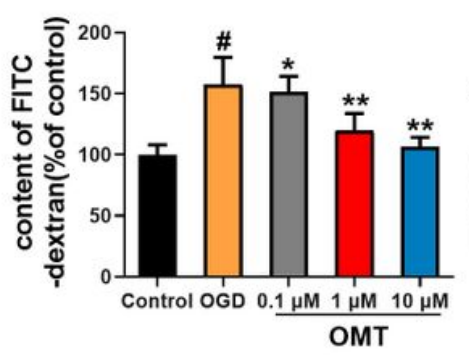

\section{Figure 4}

OMT can protect bEnd.3 cells from OGD-induced damage and increase the integrity of the BBB in vitro.

(a) Annexin-V/PI staining to detect the apoptosis of brain microvascular endothelial bEnd.3 cells after 24 $\mathrm{h}$ of reoxygenation and oxymatrine treatment. (b) Quantitative analysis of the percentage of apoptosis in each group. (c) CCK-8 assay to determine the protective effect of oxymatrine on OGD-induced decline in the viability of bEnd. 3 cells. (d) FITC-dextran leak test confirmed the protective effect of oxymatrine. (e) On the 7th day after the cells were seeded into the Transwell chamber, the TEER value was used to determine the transmembrane resistance of the monolayer cells; $n=8$ mice per group. $\# p<0.01$ versus sham group; ${ }^{* *} p<0.01,{ }^{*} p<0.05$ versus I/R group 
a
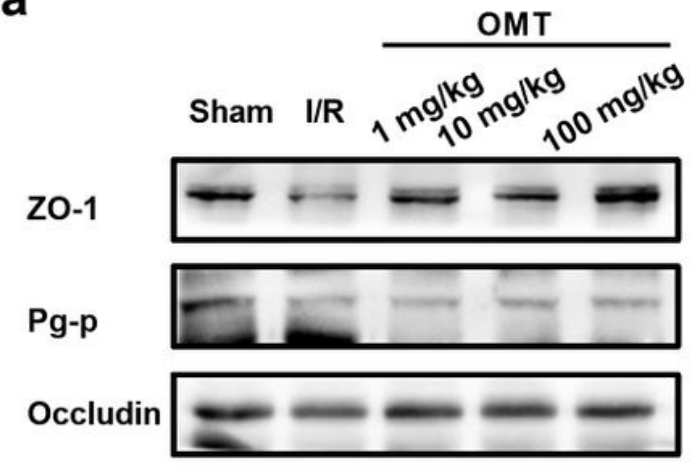

MMP-2

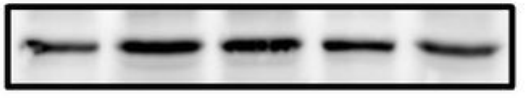

$\beta$-actin
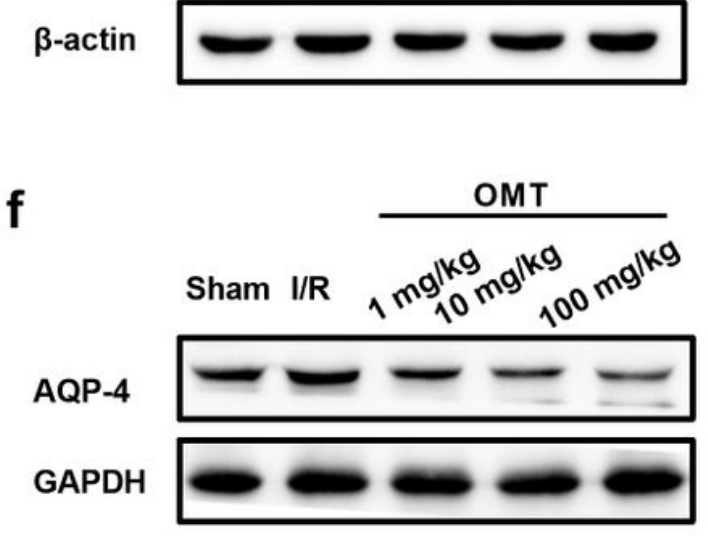

b

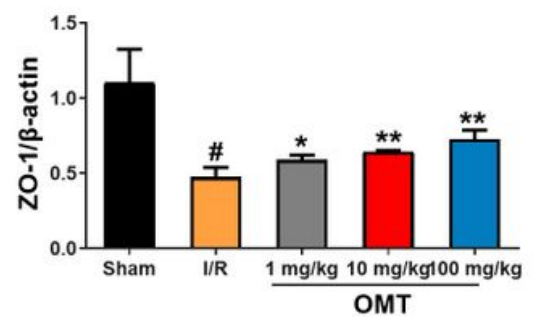

d

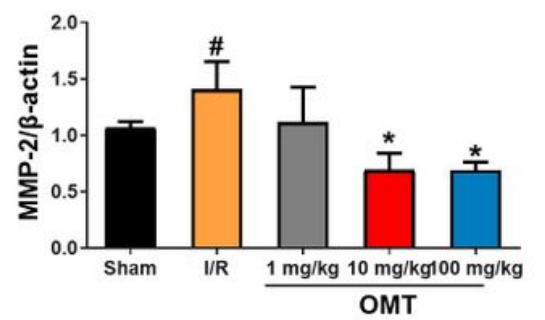

g

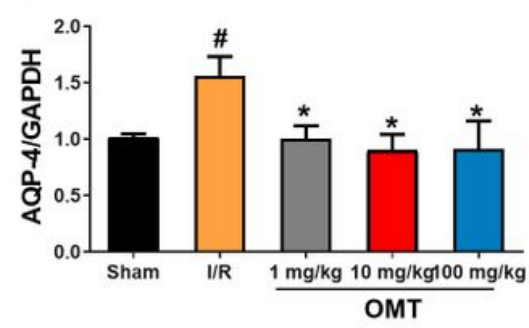

C

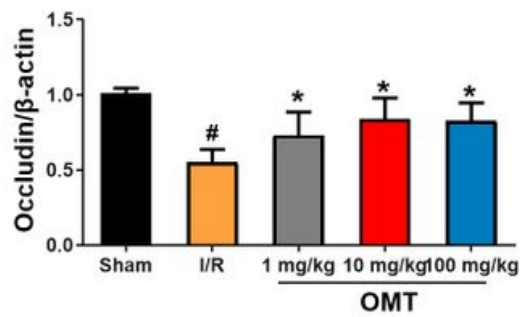

e

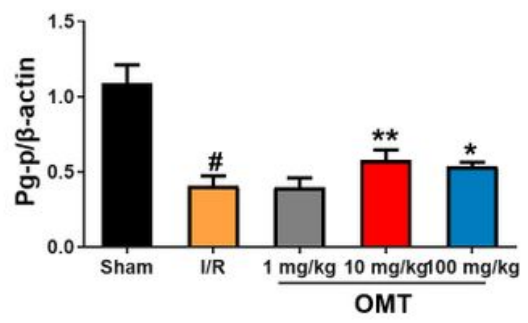

\section{Figure 5}

Oxymatrine reduces the degradation of proteins associated with BBB integrity. (a) Representative western blot analysis; (b-c) ZO-1 and Occludin, (d-e) P-gp and MMP-2, (f-g) AQP-4, and relative quantification of the band density; $\mathrm{n}=6$ mice per group. $\# \mathrm{p}<0.01$ versus sham group; ${ }^{* *} \mathrm{p}<0.01,{ }^{*} \mathrm{p}<0.05$ versus $\mathrm{I} / \mathrm{R}$ group 
a

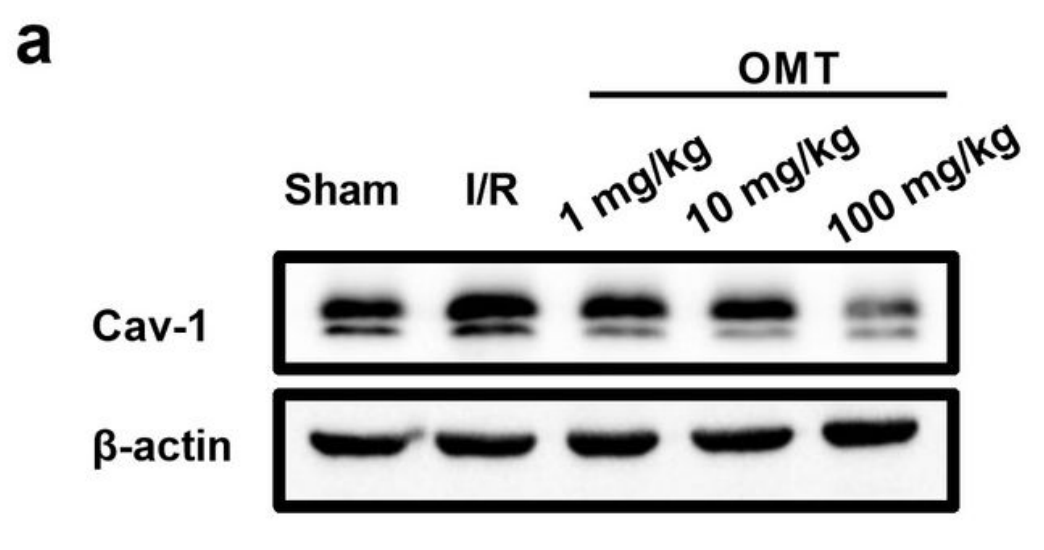

b

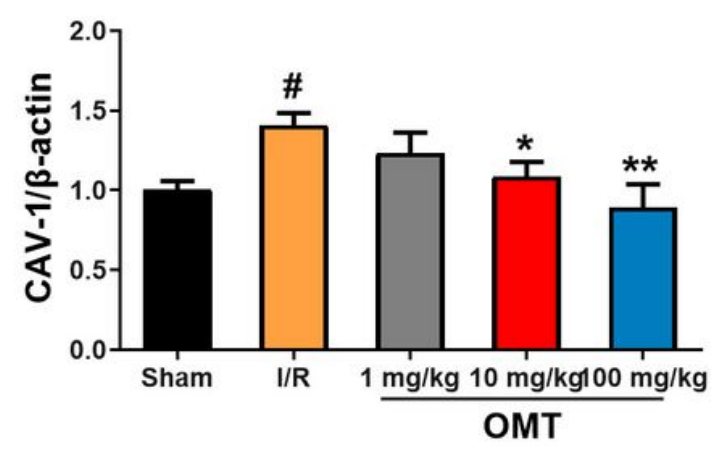

d

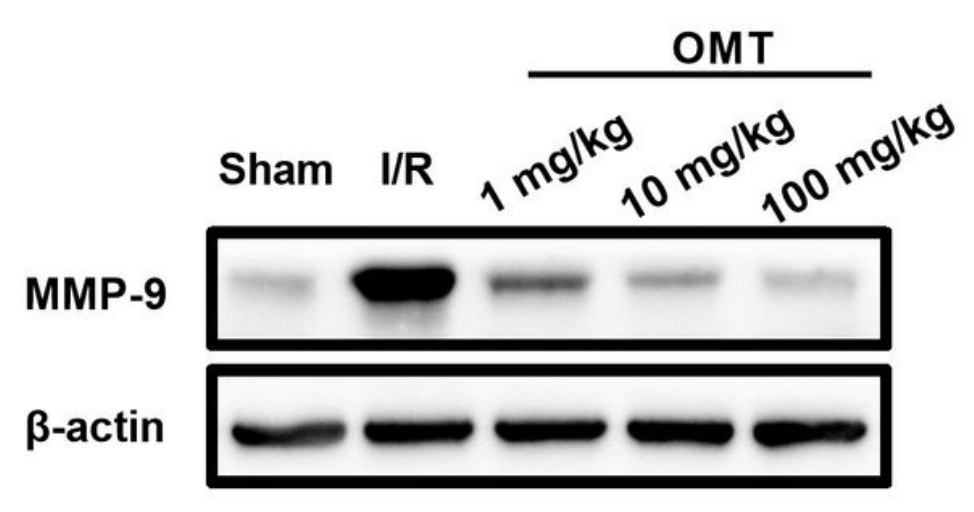

d

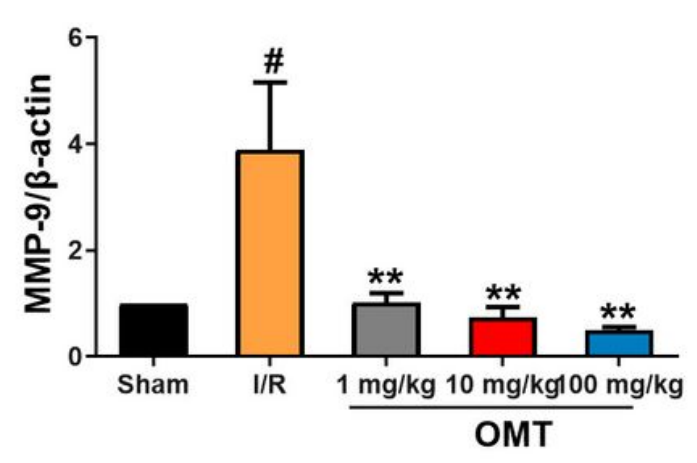

Figure 6

Effect of OMT on CAV-1/MMP-9 Signal Pathway. (a-d)Western blot analysis of the expression of CAV-1 and MMP-9, and relative quantification of the band density. OMT treatment significantly reduced Cav-1 expression, which in turn reduced the upregulation of MMP-9. $n=6$ mice per group. $\# p<0.01$ versus sham group; ${ }^{* *} \mathrm{p}<0.01,{ }^{*} \mathrm{p}<0.05$ versus $\mathrm{I} / \mathrm{R}$ group 
a

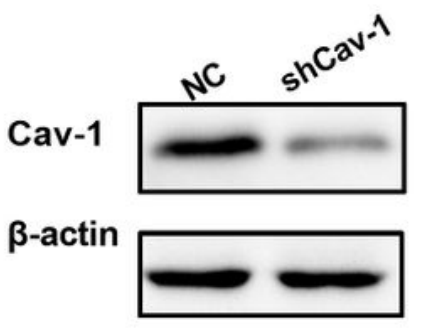

C
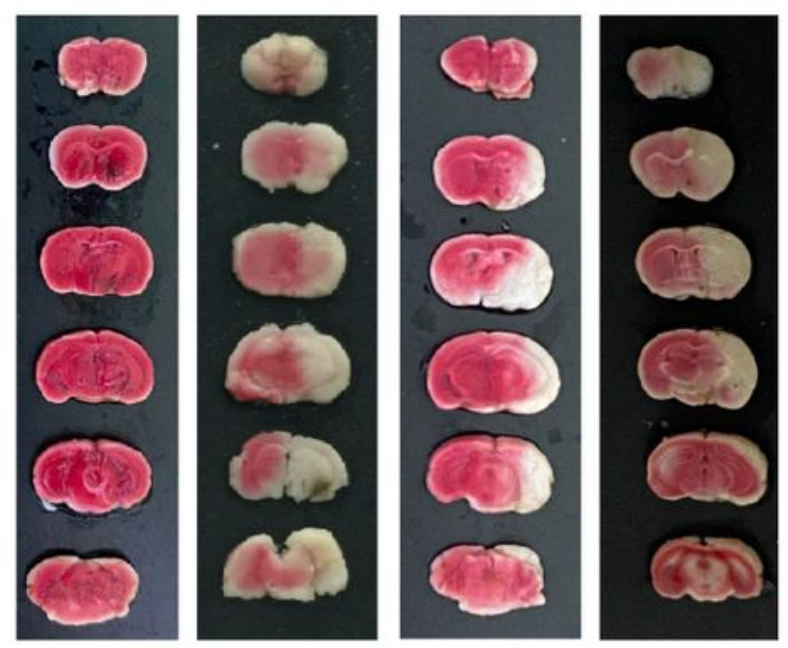

NC

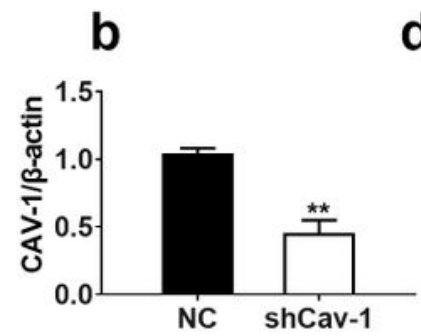

$\mathrm{NC}+\mathrm{I} / \mathrm{R}$

shCav-1 shCav-1+OMT

d

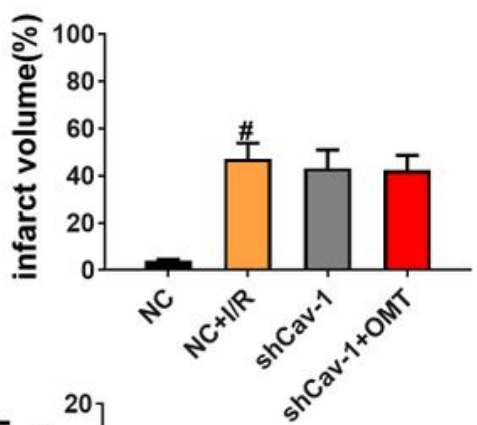

e

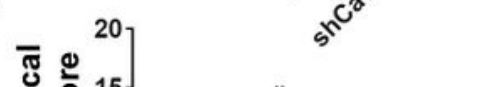

f

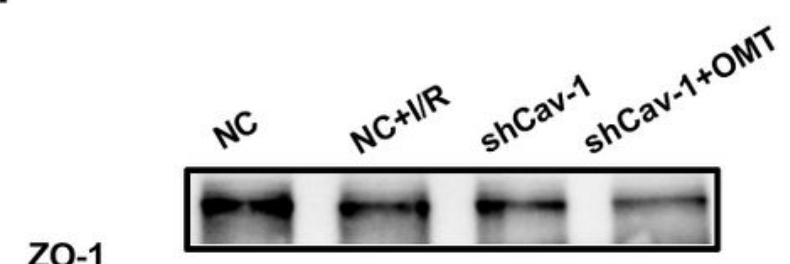

ZO-1

Occludin

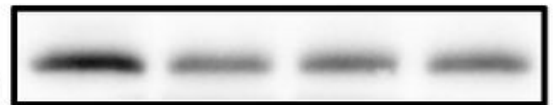

MMP-9

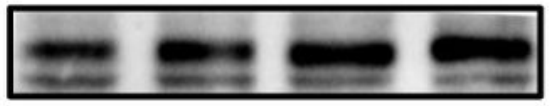

$\beta$-actin

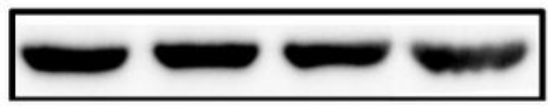

h
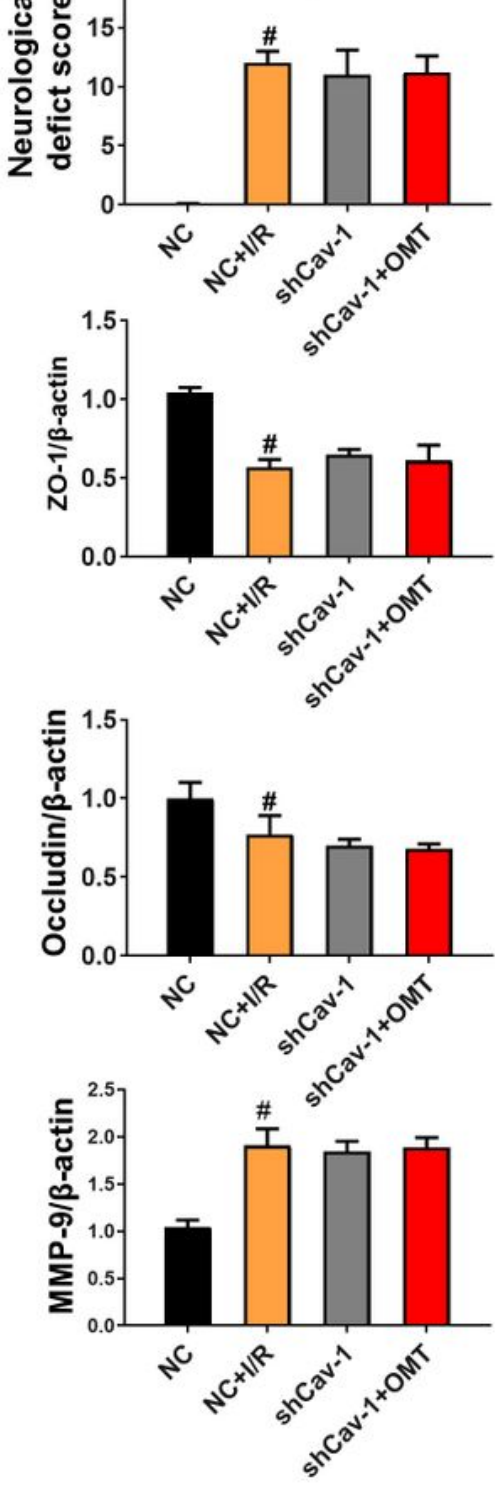

Figure 7

The brain protection of oxymatrine is weakened after CAV-1 is knocked down. (a-b) The expression of CAV-1 in the knockdown group was significantly reduced. (c-d) TTC staining results and infarct area quantification. (e) Neurological function score results. (f-i) Representative western blot analysis of MMP9, ZO-1, and Occludin, and relative quantification of the band density. $n=6$ mice per group. $\# p<0.01$ versus sham group; ${ }^{* *} \mathrm{p}<0.01,{ }^{*} \mathrm{p}<0.05$ versus $\mathrm{I} / \mathrm{R}$ group 